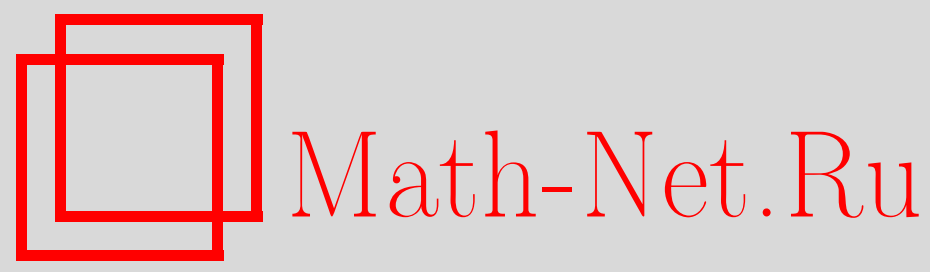

В. Т. Фоменко, Двумерные поверхности с плоской нормальной связностью в пространстве постоянной кривизны, несущие геодезические постоянной кривизны, Матем. заметки, 2000, том 68, выпуск 4, 579-586

DOI: https://doi.org/10.4213/mzm978

Использование Общероссийского математического портала Math-Net.Ru подразумевает, что вы прочитали и согласны с пользовательским соглашением http://www.mathnet.ru/rus/agreement

Параметры загрузки:

IP: 3.95 .254 .165

26 апреля 2023 г., 14:45:55

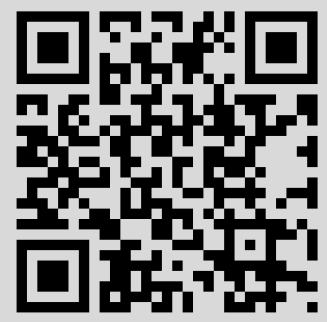




\section{ДВУМЕРНЫЕ ПОВЕРХНОСТИ С ПЛОСКОЙ НОРМАЛЬНОЙ СВЯЗНОСТЬЮ В ПРОСТРАНСТВЕ ПОСТОЯННОЙ КРИВИЗНЫ, НЕСУЩИЕ ГЕОДЕЗИЧЕСКИЕ ПОСТОЯННОЙ КРИВИЗНЫ}

\section{В. Т. Фоменко}

В работе описываются все двумерные поверхности с плоской нормальной связностью в римановом пространстве постоянной кривизны, геодезические которых имеют постоянную первую кривизну. В частности, показано, что такие поверхности являются поверхностями постоянной внутренней кривизны.

Библиографофия: 2 названия.

1. Введение. Пусть $\mathbb{R}^{n}-n$-мерное риманово пространство постоянной кривизны $R_{0}$, заданное в координатах $\left(y^{\alpha}\right)$ метрической формой $d s^{2}=a_{\alpha \beta} d y^{\alpha} d y^{\beta}$, где $a_{\alpha \beta} \in C^{2}$. Пусть $\stackrel{*}{\Gamma}_{\beta \gamma}^{\alpha}, \stackrel{*}{\nabla}_{\alpha}, \stackrel{*}{R}_{\alpha \beta, \gamma}^{\delta}-$ соответственно символы Кристоффеля, операция ковариантного дифференцирования, тензор кривизны, вычисленные относительно метрического тензора $a_{\alpha \beta}$. В дальнейшем считаем, что индексы $\alpha, \beta, \gamma, \ldots$ пробегают значения от 1 до $n$.

Пусть $F^{2}$ - двумерная поверхность в $\mathbb{R}^{n}$, заданная в окрестности каждой своей точки уравнениями

$$
y^{\alpha}=f^{\alpha}\left(x^{1}, x^{2}\right)
$$

где $f^{\alpha} \in C^{3}$.

Пусть $g_{i j}, \Gamma_{i j}^{k}, \nabla_{i}, R_{i j, k}^{l}$ - соответственно метрический тензор поверхности $F^{2}$, символы Кристоффеля, операция ковариантного дифференцирования и тензор кривизны, вычисленные относительно тензора $g_{i j}$. Индексы $i, j, k, \ldots$ принимают значения 1 и 2 . Касательную плоскость к поверхности $F^{2}$ в точке $x$ будем обозначать через $T_{x} F^{2}$. Пусть $\left\{B_{i}^{\alpha}\right\}$, где $B_{i}^{\alpha}=\partial y^{\alpha} / \partial x^{i},-$ поле реперов касательного оснащения поверхности, порожденное внутренними координатами $\left(x^{i}\right)$. Тогда метрическая форма поверхности имеет вид: $d s^{2}=g_{i j} d x^{i} d x^{j}$, где $g_{i j}=g_{\alpha \beta} B_{i}^{\alpha} B_{j}^{\beta}$.

Рассмотрим нормальное оснашения поверхности $F^{2}$, задаваемое регулярньм полем ортонормированных реперов $\left\{n_{\sigma}^{\alpha}\right\}$ в нормальном расслоении $N F^{2}$ поверхности $F^{2}$. В дальнейшем индексы $\sigma, \tau, \lambda, \ldots$ принимают значения от 3 до $n$. Пусть $g_{\sigma \tau}, \Gamma_{i \tau}^{\sigma}, \nabla^{\perp}$, $R_{i j, \sigma}^{\tau}-$ соответственно метрический тензор слоя $N_{x} F^{2}$ нормального расслоения $N F^{2}$ в

Работа выполнена при финансовой поддержке Российского фонда фундаментальных исследований, грант № 99-01-00814. 
точке $x$, коэффициенты нормальной связности, операция ковариантного дифференцирования и тензор кривизны, вычисленные относительно коэффициентов $\Gamma_{i \tau}^{\sigma}$.

Для заданного поля ортогональных реперов $\left\{n_{\sigma}^{\alpha}\right\}$ имеем

$$
g_{\sigma \tau}=a_{\alpha \beta} n_{\sigma}^{\alpha} n_{\tau}^{\beta}, \quad \Gamma_{\sigma, i \tau}=a_{\alpha \beta} n_{\sigma}^{\alpha} \stackrel{\nabla}{i}_{i} n_{\tau}^{\beta}, \quad g^{\sigma \lambda} \Gamma_{\sigma, i \tau}=\Gamma_{i \tau}^{\lambda}, \quad\left\|g^{\sigma \lambda}\right\|=\left\|g_{\sigma \lambda}\right\|^{-1}
$$

Линейные формы $\omega_{\sigma \tau}=\Gamma_{\sigma, i \tau} d x^{i}$ называют линейными формами кручения поверхности $F^{2}$.

Обозначим через $I I_{\sigma}=b_{\sigma i j} d x^{i} d x^{j}$ вторую квадратичную форму поверхности $F^{2}$ относительно нормали $n_{\sigma}^{\alpha}$, где $b_{\sigma i j}$ вычисляются по формуле $b_{\sigma i j}=a_{\alpha \beta} n_{\sigma}^{\alpha} \stackrel{\nabla}{i}_{i} B_{j}^{\beta}$.

На поверхности $F^{2}$ имеют место формулы Гаусса-Вейнгартена

$$
\bar{\nabla}_{j} B_{i}^{\alpha}=b_{i j}^{\sigma} n_{\sigma}^{\alpha}, \quad \bar{\nabla}_{j} n_{\sigma}^{\alpha}=-b_{\sigma j}^{k} B_{k}^{\alpha}
$$

где $\bar{\nabla}=\stackrel{*}{\nabla} \oplus \nabla \oplus \nabla^{\perp}$ обозначает ковариантное дифференцирование в $\mathbb{R}^{n}$ вдоль $F^{2}$ в смысле Ван дер Вардена-Бортолотти, определяемые формулами

$$
\begin{aligned}
& \bar{\nabla}_{j} B_{i}^{\alpha}=\partial_{j} B_{i}^{\alpha}-\Gamma_{j i}^{k} B_{k}^{\alpha}+\stackrel{\Gamma}{\Gamma}_{\beta \gamma}^{\alpha} B_{j}^{\beta} B_{i}^{\gamma}, \\
& \bar{\nabla}_{j} n_{\sigma}^{\alpha}=\partial_{j} n_{\sigma}^{\alpha}-\Gamma_{j \sigma}^{\tau} n_{\tau}^{\alpha}+\stackrel{\Gamma}{\Gamma}_{\beta \gamma}^{\alpha} B_{j}^{\beta} n_{\sigma}^{\gamma} .
\end{aligned}
$$

Условия интегрируемости системы (1), (2) в римановом пространстве определяются уравнениями Гаусса-Кодацци-Риччи:

$$
\begin{aligned}
R_{i j, k l} & =g_{\sigma \tau} b_{[i \underline{k}}^{\sigma} b_{j] l}^{\tau}+\stackrel{*}{R}_{\alpha \beta, \gamma \delta} B_{i}^{\alpha} B_{j}^{\beta} B_{k}^{\gamma} B_{i}^{\delta}, \\
\bar{\nabla}_{[k} b_{\underline{\sigma} i] j} & =\stackrel{*}{R}_{\alpha \beta, \gamma \delta} B_{i}^{\alpha} B_{j}^{\gamma} B_{k}^{\delta} n_{\sigma}^{\beta}, \\
R_{i j, \sigma \tau} & =g^{l h} b_{\sigma\left[i \underline{i}{ }^{\prime}\right.} b_{\tau j] h}+\stackrel{*}{R}_{\alpha \beta, \gamma \delta} B_{i}^{\alpha} B_{j}^{\beta} n_{\sigma}^{\alpha} n_{\tau}^{\beta} .
\end{aligned}
$$

Так как в римановом пространстве $\mathbb{R}^{n}$ постоянной кривизны $R_{0}$ тензор кривизны имеет строение:

$$
R_{\alpha \beta, \gamma \delta}=R_{0}\left(a_{\alpha \gamma} a_{\beta \delta}-a_{\alpha \delta} a_{\beta \gamma}\right),
$$

то последняя система для двумерной поверхности принимает вид

$$
\begin{aligned}
R_{12,21} & =g_{\sigma \tau} b_{1[1}^{\sigma} b_{2] 2}^{\tau}+R_{0} g \\
\bar{\nabla}_{[1} b_{2] 2}^{\sigma} & =0, \quad \bar{\nabla}_{[1} b_{2] 1}^{\sigma}=0 \\
R_{12,34} & =b_{3 l[1} b_{\underline{42} 2] h} g^{l h}
\end{aligned}
$$

где $\left\|g^{l h}\right\|=\left\|g_{l h}\right\|^{-1}, g=\operatorname{det}\left\|g_{i j}\right\|$. Известно, что решение системы (3)-(5) относительно $g_{i j}, b_{\sigma i j}, \Gamma_{i \tau}^{\sigma}$ порождает в пространстве $\mathbb{R}^{n}$ постоянной кривизны поверхность $F^{2}$, для которой эти функции служат соответственно метрическим тензором, тензором вторых квадратичных форм и коэффициентами нормальной связности; при этом поверхность определена с точностью до движения в $\mathbb{R}^{n}$. 
Если $R_{i j, k}^{l} \equiv 0$, то метрика поверхности назьвается плоской. Поверхности $F^{2}$ с плоской метрикой характеризуются тем свойством, что на них и только на них можно выбрать внутренние координаты $\left(x^{i}\right)$ так, что $\Gamma_{j k}^{i} \equiv 0$. Метрика поверхности $F^{2}$ в этом случае может быть записана в виде $d s^{2}=\left(d x^{1}\right)^{2}+\left(d x^{2}\right)^{2}$. Если $R_{i j, \sigma}^{\tau} \equiv 0$, то нормальная связность поверхности $F^{2}$ назьвается плоской. Поверхности с плоской нормальной связностью характеризуются тем свойством, что на них и только на них существует нормальное оснащение с полем ортонормированных реперов $\left\{n_{\sigma}^{\alpha}\right\}$, для которого $\Gamma_{i \tau}^{\sigma} \equiv 0$.

Пусть $L: x^{i}=x^{i}(s)$ - кривая на поверхности, заданная уравнениями, отнесенными к натуральному параметру. Пусть $k^{\alpha}-$ вектор первой кривизны кривой $L$ в $\mathbb{R}^{n}$. Составляющая $k_{g}^{\alpha}$ вектора $k^{\alpha}$ на касательную плоскость $T_{x} F^{2}$ назьвается вектором геодезической кривизны кривой $L$ в точке $x$, а его длина $k_{g}$ - геодезической кривизной кривой $L$. Если $k_{g}=0$ вдоль $L$, то линия $L$ называется геодезической на $F^{2}$ и обозначается через $L_{g}$. Составляющая $k_{n}^{\alpha}$ вектора кривизны $k^{\alpha}$ на нормальную плоскость $N_{x} F^{2}$ назьвается вектором нормальной кривизны поверхности $F^{2}$ в точке $x$ по направлению $t^{i}=d x^{i} /(d s)$, а его длина $k_{n}=\sqrt{a_{\alpha \beta} k_{n}^{\alpha} k_{n}^{\beta}}-$ нормальной кривизной $F^{2}$ в точке по направлению $t^{\alpha}$. Известно, что $k_{n}$ не зависит от выбора кривой $L$ и является функцией точки $x$ и направления $t^{\alpha}$. Известна формула вычисления $k_{n}$ :

$$
k_{n}=\sqrt{g_{\sigma \tau} b_{i j}^{\sigma} b_{k l}^{\tau} t^{i} t^{j} t^{k} t^{l}}
$$

Конец вектора $k_{n}^{\alpha}$ определяет точку $P$ в нормальной плоскости $N_{x} F^{2}$. При изменении направления $t^{\alpha}$ в $T_{x} F^{2}$ точка $x$ описывает в $N_{x} F^{2}$ кривую, которая назьвается $u н \partial u$ катрисой нормальной кривизны поверхности в точке $x$. Индикатриса нормальной кривизны поверхности $F^{2}$ в точке $x$ есть эллипс. Эллипс нормальной кривизны вырождается в отрезок (или точку) тогда и только тогда, когда поверхность $F^{2}$ имеет плоскую нормальную связность. В этом и только этом случае на поверхности $F^{2}$ существует координатная сеть, для которой $g_{12}=0, b_{\sigma 12}=0$ при любом выборе $\left\{n_{\sigma}^{\alpha}\right\}$. Направления координатньх линий в этом случае назьвают главными направлениями на поверхности, а нормальные кривизны поверхности по главньм направлениям называют главными кривизнами поверхности и обозначают $k_{n i}$. Если $k_{n 1}=k_{n 2}=0$ в точке $x$, то точка $x$ называется точкой уплощения, в случае $k_{n 1}=0, k_{n 2} \neq 0$ точка назьвается параболической точкой. Длина $E=E(x)$ отрезка вырожденного эллипса нормальной кривизны в точках назьвается эйлеровой разностью поверхности в точке $x$. Если в точке $x$ имеем $E=0, c^{2}=k_{n 1}^{2}+k_{n 2}^{2} \neq 0$, то точка $x$ назьвается омбилической точкой с радиусом $1 /$. Очевидно, что в омбилической точке эллипс нормальной кривизны вырождается в точку, не совпадающую с точкой $x$. Точка называется аксиальной, если нормальное оснащение можно выбрать таким, чтобы носитель вектора нормали $n_{3}^{\alpha}$ проходил через точку $x$.

\section{2. Формулировка результата.}

ТеоремА. Для того чтобы на двумерной поверхности $F^{2}$ класса $C^{3}$ с плоской нормальной связностью в пространстве $\mathbb{R}^{n}$ постоянной кривизны $R_{0}$ каждая геодезическая имела постоянную первую кривизну, необходимо и достаточно, чтобь поверхность принадлежсала одному из следующ, видов поверхностей:

1) вполне геодезические поверхности; 
2) поверхности постоянной внутренней кривизны $K$, состоящие из омбилических точек радиуса $1 / \sqrt{K-R_{0}}$, при условии $K>R_{0}$;

3) поверхности нулевой внутренней кривизны $K \equiv 0$ с постоянными ненулевыми главными кривизнами $k_{n 1}=c_{1}>0, k_{n 2}=c_{2}>0$ и постоянной эйлеровой разностью Е при условии $c_{1}^{2}+c_{2}^{2}>-2 R_{0}$;

4) поверхности нулевой внутренней кривизны $K \equiv 0$ с аксиальными точками и постоянными главными кривизнами $k_{n 1}=c_{1} \geqslant 0, k_{n 2}=c_{2}>0$ при условии $c_{1} c_{2}=-R_{0}$.

Каждьй из указанных в теореме видов поверхностей является непустым при выполнении отмеченных условий. Укажем некоторые примеры поверхностей каждого из перечисленных видов.

$\mathrm{K}$ первому виду следует отнести плоскость Лобачевского кривизны $R_{0}$ при $R_{0}<0$; плоскости в евклидовом пространстве при $R_{0}=0$ и сферы кривизны $R_{0}$ при $R_{0}>0$.

Ко второму виду поверхностей относятся, в частности, сферы, орисферы и эквидистанты в пространстве Лобачевского.

$\mathrm{K}$ третьему виду поверхностей относится, например, тор Клиффорда $S^{1} x S^{1}$ в $E^{n}$. Однако, этот вид поверхностей не исчерпывается торами Клиффорда. В самом деле, рассмотрим в пространстве $\mathbb{R}^{n}$ кривизны $R_{0}$ поверхность $F_{k}^{2}$, задаваемую следуюшими основньми формами:

$$
\begin{aligned}
d s^{2} & =d u^{2}+d v^{2} \\
I I_{3} & =a^{2} d u^{2}-b^{2} d v^{2}, \\
I I_{4} & =h\left(d u^{2}+d v^{2}\right), \quad a^{2} b^{2}-h^{2}=-R_{0}, \\
I I_{\sigma} & \equiv 0, \quad \sigma \geqslant 5, \\
\omega_{35} & =\varphi(u) d u+\phi(v) d v, \quad \omega_{34} \equiv 0, \quad \omega_{3 \sigma} \equiv 0, \quad \sigma>5 ; \\
\omega_{45} & =c \omega_{35}, \quad c=\text { const }, \quad \omega_{4 \sigma} \equiv 0, \quad \sigma>5 ; \\
\omega_{\sigma \tau} & \equiv 0, \quad \sigma \geqslant 5, \quad \tau \geqslant 6,
\end{aligned}
$$

где $\phi, \varphi$ - дифференцируемые функции своих аргументов, $a>0, b>0, c, h$ - заданные постоянные. Так как уравнения Гаусса-Кодацци-Риччи для этих форм вьполнены, то в $\mathbb{R}^{n}$ существует поверхность $F_{k}^{2}$, для которой эти формулы являются основными формулами. Такую поверхность назовем тором Клиффорда с кручением. Отметим, что поворотом репера $\left\{n_{\sigma}^{\alpha}\right\}$ можно добиться того, чтобы все линейные формы кручения $\omega_{\sigma \tau}$ тождественно обращались в нуль. Но тогда в новом репере квадратичные формы $I I_{\sigma}$, $\sigma \geqslant 5$, не будут тождественно равны нулю.

Отметим, что для указанных в теореме поверхностей третьего вида в евклидовом пространстве $E^{n}$ индикатриса нормальной кривизны в $N_{x} F^{2}$ просматривается из точки $x \in F^{2}$ под прямым углом. Общее описание поверхностей указанного вида можно найти в $[1$, разд. III, п. 14 , с. 164-169].

K четвертому виду поверхностей относится, например, круглый цилиндр с кручени- 
ем в $E^{n}$ (см., например, [2]), определяемый следуюшими основными формами:

$$
\begin{aligned}
d s^{2} & =d u^{2}+d v^{2}, \\
I I_{3} & =\varphi_{1}(u) d u^{2}, \quad I I_{\sigma} \equiv 0, \quad \sigma \geqslant 4, \\
\omega_{\sigma \tau} & =\varphi_{\sigma-2}(u) d u, \quad \tau=\sigma-1, \quad \sigma \geqslant 4, \\
\omega_{\sigma \tau} & \equiv 0, \quad \tau \neq \sigma-1,
\end{aligned}
$$

где $\varphi_{\sigma-2}, \sigma \geqslant 3,-$ заданные дифференцируемые функции аргумента $u$. Уравнение поверхности $F_{c}^{2}$, определяемой указанными формами в $E^{n}$, имеет вид $r=\rho(u)+v a+b$, где $\rho=\rho(u)$ - уравнения кривой $(l)$, лежащей в некотором $E^{n-1}$, и отнесенное к натуральному параметру $u ; a$ - вектор нормали к $E^{n-1}, b$ - некоторьй постоянньй вектор.

$\Phi$ ункции $\varphi_{1}, \varphi_{2}, \ldots, \varphi_{n-2}$ являются кривизнами кривой $(l)$.

Отметим, что нормальное оснащение поверхности $F_{c}^{2}$ задается $(n-2)$-гранником $\Phi$ рене кривой $(l)$, параллельно перенесенньм из точек кривой вдоль образующих по всей поверхности $F_{c}^{2}$. Поверхность $F_{c}^{2}$ при $\varphi_{1}(u)=k_{1}=$ const назьвают круглым цилиндром с кручением.

ЗАмЕчАниЕ. Предположение, что нормальная связность поверхности является плоской, существенно. Действительно, на поверхности Веронезе $V^{2}$ в $E^{5}$ геодезические имеют постоянную кривизну, однако нормальная связность на $V^{2}$ не является плоской.

3. Доказательство теоремы. Пусть $L_{g}$-геодезическая на поверхности $F^{2}$. Условие постоянства первой кривизны кривой $L_{g}$ в $\mathbb{R}^{n}$ эквивалентно условию постоянства вдоль $L_{g}$ нормальной кривизны $k_{n}\left(x, t^{\alpha}\right)$ :

$$
\bar{\nabla}_{i} k_{n}\left(x, t^{\alpha}\right) t^{i}=0
$$

где $t^{i}=d x^{i}(s) / d s$. Из формулы (7) в силу (6) находим

$$
g_{\sigma \tau} b_{i j}^{\sigma} \bar{\nabla}_{k} b_{l p}^{\tau} t^{i} t^{j} t^{k} t^{l} t^{p}=0 .
$$

Так как $t^{1}, t^{2}$ в формуле (8) произвольны, то это соотношение имеет место, если коэффициенты его левой части равны нулю. Учитьвая формулы Кодацши (4), из (8) находим:

$$
\begin{aligned}
& g_{\sigma \tau} b_{i i}^{\sigma} \bar{\nabla}_{i} b_{i i}^{\tau}=0, \\
& g_{\sigma \tau} b_{i i}^{\sigma} \bar{\nabla}_{j} b_{i i}^{\tau}=0, \\
& 3 g_{\sigma \tau} b_{i i}^{\sigma} \bar{\nabla}_{i} b_{j j}^{\tau}+g_{\sigma \tau} b_{j j}^{\sigma} \bar{\nabla}_{i} b_{i i}^{\tau}=0 .
\end{aligned}
$$

Для поверхностей $F^{2}$ с плоской нормальной связностью опишем все решения систем (3)$(5),(9)-(11)$ относительно $g_{i j}, b_{i j}^{\sigma}, \Gamma_{i \tau}^{\sigma}$. Примем в качестве координатных линий на $F^{2}$ в окрестности некоторой ее точки сеть линий кривизны. Тогда для любого регулярного нормального оснащения $F^{2}$ в окрестности этой точки с полем реперов $\left\{n_{\sigma}^{\alpha}\right\}$ будем иметь $g_{12}=0, b_{\sigma 12}=0$. При этом можно считать, что $g_{i j} \in C^{1}, b_{\sigma i j} \in C^{1}, R_{12,12} \in C$. Выберем нормальное оснащение поверхности $F^{2}$ таким образом, чтобы $\Gamma_{i \tau}^{\sigma}=0$ в окрестности рассматриваемой точки. Тогда система уравнений (9) приводится к виду:

$$
\partial_{i} \ln g_{\sigma \tau} b_{i i}^{\sigma} b_{i i}^{\tau}=\partial_{i} \ln g_{i i}^{2}
$$


а из системы (10) находим

$$
\partial_{i} \ln g_{\sigma \tau} b_{j j}^{\sigma} b_{j j}^{\tau}=\partial_{i} \ln g_{j j}^{2}, \quad i \neq j
$$

Система уравнений (12), (13) интегрируется в явном виде; в результате из (12), (13) получаем

$$
g_{\sigma \tau} b_{i i}^{\sigma} b_{i i}^{\tau}=c_{i}^{2} g_{i i}^{2}
$$

где $c_{i}$ - произвольные постоянные. Полагая $\Gamma_{i \tau}^{\sigma} \equiv 0$, перепишем систему (11). Имеем

$$
3 g_{\sigma \tau} b_{i i}^{\sigma} \partial_{i} b_{j j}^{\tau}+g_{\sigma \tau} b_{j j}^{\sigma}\left(\partial_{i} b_{i i}^{\tau}-b_{i i}^{\tau} \partial_{i} \ln \left(g_{i i} g_{j j}^{3}\right)\right)=0 .
$$

Воспользуемся уравнениями Кодацщи (4), которые запишем в виде:

$$
2 \partial_{i} b_{j j}^{\sigma}-b_{j j}^{\sigma} \partial_{i} \ln g_{22}-\frac{b_{i i}^{\sigma}}{g_{i i}} \partial_{i} g_{j j}=0
$$

Умножим (15) на $g_{\sigma \tau} b_{j j}^{\tau}$ и просуммируем по $\sigma$. Имеем

$$
\partial_{i}\left(g_{\sigma \tau} b_{j j}^{\sigma} b_{j j}^{\tau}\right)-g_{\sigma \tau} b_{j j}^{\sigma} b_{j j}^{\tau} \partial_{i} \ln g_{j j}-g_{\sigma \tau} \frac{b_{i i}^{\sigma}}{g_{i i}} b_{j j}^{\tau} \partial_{i} g_{j j}=0
$$

В силу уравнения Гаусса $(3)$, которое в нашем случае записьвается в виде $\left(K-R_{0}\right) g_{i i} g_{j j}$ $=g_{\sigma \tau} b_{i i}^{\sigma} b_{j j}^{\tau}, i \neq j$, из $(14),(16)$ находим соотношение

$$
\partial_{i} g_{j j}\left(c_{i}^{2}-\left(K-R_{0}\right)\right)=0, \quad i \neq j .
$$

Исследуем соотношение (17).

Случай 1. Пусть $\partial_{i} g_{j j}=0, i \neq j$. Это означает, что $g_{11}=g_{11}\left(u^{1}\right), g_{22}=g_{22}\left(u^{2}\right)$. Переходя к новым координатам $\bar{x}^{i}$ по формулам

$$
\bar{x}^{1}=\int \sqrt{g_{11}\left(x^{1}\right)} d x^{1}, \quad \bar{x}^{2}=\int \sqrt{g_{22}\left(x^{2}\right)} d x^{2},
$$

получим, что координатная сеть не изменилась, но в новых координатах $\bar{g}_{11}=\bar{g}_{22}=1$. Будем считать, что в координатах $x^{i}$ имеем $g_{11}=g_{22}=1$. Из соотношений $(15)$ находим $\partial_{i} b_{j j}^{\sigma}=0, i \neq j$. Это означает, что

$$
b_{11}^{\sigma}=b_{11}^{\sigma}\left(x^{1}\right), \quad b_{22}^{\sigma}=b_{22}^{\sigma}\left(x^{2}\right) .
$$

Будем рассматривать $\left(b_{11}^{\sigma}\right)$ и $\left(b_{22}^{\sigma}\right)$ как векторы $\vec{k}_{1 n}$ и $\vec{k}_{2 n}$ в $N_{x} F^{2}$. Тогда уравнение (14) можно записать в виде скалярных произведений: $\left(\vec{k}_{1 n}, \vec{k}_{1 n}\right)=c_{1}^{2},\left(\vec{k}_{2 n}, \vec{k}_{2 n}\right)=c_{2}^{2}$. Отсюда следует, что

$$
b_{11}^{\sigma}=c_{1} \cos \alpha^{\sigma}\left(x^{1}\right), \quad b_{22}^{\sigma}=c_{2} \cos \beta^{\sigma}\left(x^{2}\right),
$$

где $\cos \alpha^{\sigma}, \cos \beta^{\sigma}$ - соответственно направляющие косинусы векторов $\vec{k}_{1 n}, \vec{k}_{2 n}$ в $N_{x} F^{2}$. В силу (19) из уравнений (18) находим:

$$
g_{\sigma \tau} b_{i i}^{\sigma}\left(x^{i}\right) \partial_{j} b_{j j}^{\tau}\left(x^{j}\right)=0, \quad i \neq j
$$


Это означает, что должны выполняться соотношения:

$$
\begin{aligned}
& c_{1} c_{2} g_{\sigma \tau} \cos \beta^{\sigma}\left(x^{2}\right) \sin \alpha^{\tau}\left(x^{1}\right) \partial_{1} \alpha^{\tau}\left(x^{1}\right)=0, \\
& c_{1} c_{2} g_{\sigma \tau} \cos \alpha^{\sigma}\left(x^{1}\right) \sin \beta^{\tau}\left(x^{2}\right) \partial_{2} \beta^{\tau}\left(x^{2}\right)=0 .
\end{aligned}
$$

Эти соотношения выполняются тождественно в силу уравнения Гаусса, которое записьвается в виде:

$$
c_{1} c_{2} g_{\sigma \tau} \cos \alpha^{\sigma} \cos \beta^{\tau}=-R_{0}
$$

Исследуем уравнение (20).

а) Пусть $c_{1}=0$. Из $(20)$ следует, что $R_{0}=0$, и потому $\mathbb{R}^{n}=E^{n}$, где $E^{n}-n$-мерное евклидово пространство. Тогда $b_{11}^{\sigma} \equiv 0, b_{22}^{\sigma} \equiv c_{2} \cos \beta^{\sigma}\left(x^{2}\right)$. Это означает, что индикатриса кривизны есть отрезок с началом в точке $x, x \in F^{2}$, т. е. $x$-параболическая точка. Повернем $\left\{\vec{n}_{\sigma}\right\}$ в положение $\left\{\vec{n}_{\sigma}^{*}\right\}$ так, чтобы $\vec{n}_{3}^{*}$ совпал с направлением индикатрисы. Тогда $d s^{2}=\left(d x^{1}\right)^{2}+\left(d x^{2}\right)^{2}$,

$$
I I_{3}=c_{2}\left(d x^{2}\right)^{2}, \quad I I_{\sigma} \equiv 0, \quad \sigma=3, \ldots, n, \quad \omega_{\sigma \tau}=\Gamma_{2 \sigma \tau} d x^{2}
$$

где $\Gamma_{2 \sigma \tau}$, вообще говоря, отличны от нуля. Как показано в пункте 2, к таким поверхностям относится кругльй цилиндр с кручением при $c_{2}=$ const $\neq 0$.

В случае $c_{2}=0$ имеем двумерную плоскость в $E^{n}$, ибо можно выбрать поле реперов $\left\{\vec{n}_{\sigma}\right\}$ с условием $b_{\sigma i j}=0, \Gamma_{i \sigma}^{\tau}=0$.

б) Пусть $c_{1} \neq 0, c_{2} \neq 0, R_{0}=0$. Тогда из уравнения Гаусса находим $\left(\vec{k}_{1 n}, \vec{k}_{2 n}\right)=0$. Это означает, что индикатриса нормальной кривизны просматривается под прямьм углом, при этом длина перпендикуляра, опущенного из точки $x$ на индикатрису, постоянна в окрестности рассматриваемой точки. Выберем $\left\{\vec{n}_{\sigma}\right\}$ таким образом, чтобы носители $\vec{n}_{3}$ и $\vec{n}_{4}$ лежали в плоскости, проходящей через точку $x$ и индикатрису, а их носители были параллельны и ортогональны индикатрисе соответственно. Тогда основные формы поверхности $F^{2}$ примут вид:

$$
\begin{aligned}
d s^{2} & =\left(d x^{1}\right)^{2}+\left(d x^{2}\right)^{2} \\
I I_{3} & =a_{1}\left(d x^{1}\right)^{2}+a_{2}\left(d x^{2}\right)^{2}, \\
I I_{4} & =b\left(\left(d x^{1}\right)^{2}+\left(d x^{2}\right)^{2}\right), \quad b=\mathrm{const}, \\
a_{1}^{2} & =c_{1}^{2}-b^{2}, \quad a_{2}^{2}=c_{2}^{2}-b^{2}, \\
I I_{\sigma} & \equiv 0, \quad \sigma \geqslant 5
\end{aligned}
$$

при этом линейные формы кручения $\omega_{\sigma \tau}=\Gamma_{\sigma i \tau} d x^{i}$, вообще говоря, не равны нулю тождественно. Это означает, что эйлерова разность $E$ постоянна на $F^{2}$, отлична от нуля (в общем случае) и рассматриваемую поверхность можно считать обобщением тора Клиффорда в $E^{n}$. Пример такой поверхности дан в пункте 2 (тор Клиффорда $F_{k}^{2}$ с кручением).

в) Пусть $c_{1} \neq 0, c_{2} \neq 0, R_{0} \neq 0$. Тогда угол $\varphi$, под которьм просматривается отрезок индикатрисы нормальной кривизны из точки $x$, постоянен в окрестности точки $x$. В самом деле, выберем $\left\{\vec{n}_{\sigma}\right\}$ таким образом, чтобы носители $\vec{n}_{3}$ и $\vec{n}_{4}$ лежали в одной плоскости с точкой $x$ и отрезком индикатрисы и были соответственно параллельны и 
ортогональны индикатрисе. Тогда вторые квадратичные формы $I I_{\sigma}$ поверхности $F^{2}$ примут вид:

$$
\begin{aligned}
& I I_{3}=b_{311}\left(d x^{1}\right)^{2}+b_{322}\left(d x^{2}\right)^{2}, \\
& I I_{4}=b_{411}\left(\left(d x^{1}\right)^{2}+\left(d x^{2}\right)^{2}\right), \\
& I I_{\sigma} \equiv 0, \quad \sigma \geqslant 5 .
\end{aligned}
$$

Если точка $x$ - аксиальная, то $b_{411}=0$ и тогда $I I_{4} \equiv 0$. Из уравнения Гаусса имеем $b_{311} b_{322}=-R_{0}$. Из (14) следует, что $b_{311}=c_{1}=$ const, $b_{322}=c_{2}=$ const, при этом $c_{1} c_{2}=-R_{0}$. Если точка $x$ не является аксиальной, то из уравнения Гаусса следует, что угол между $\vec{k}_{1 n}$ и $\vec{k}_{2 n}$, т. е. угол, под которьм просматривается индикатриса кривизны, постоянен в окрестности точки и вычисляется по формуле $\cos \varphi=\left(-R_{0}\right) /\left(c_{1} c_{2}\right)$. Это означает, что эйлерова разность поверхности $F^{2}$ постоянна: $E=\sqrt{c_{1}^{2}+c_{2}^{2}+2 R_{0}}$.

Случай 2. Пусть $c_{i}^{2}-\left(K-R_{0}\right)=0, i=1,2$. Тогда $c_{1}^{2}=c_{2}^{2}$. Это означает, что главные кривизны $k_{1 n}$ и $k_{2 n}$ поверхности $F^{2}$ совпадают, $k_{1 n}=k_{2 n}=c_{1}$. Из уравнения Гаусса следует, что $\left(\vec{k}_{1 n}, \vec{k}_{2 n}\right)=K-R_{0}$, т. е. $c_{1} c_{2} \cos \left(\vec{k}_{1 n}, \vec{k}_{2 n}\right)=c_{1}^{2}$. Это означает, что векторы $\vec{k}_{1 n}$ и $\vec{k}_{2 n}$ коллинеарны и сонаправлены. Следовательно, индикатриса кривизны есть точка, поэтому все точки поверхности являются омбилическими радиуса $1 / c_{1}$. Отсюда вытекает, что $K=$ const, $K=c_{1}^{2}+R_{0}$, где $c_{1}-$ произвольно заданное положительное число, что и доказьвает теорему.

\section{СПИСОК ЦИТИРОВАННОЙ ЛИТЕРАТУРЫ}

[1] Эйзенхарт Л. Т. Риманова геометрия. М.: Гос. изд. ИН., 1948.

[2] Рашевский П. К. Риманова геометрия и тензорный анализ. М.: Наука, 1964.

Таганрогский государственный педагогический институт 